Research Article

\title{
Relationship between Macrovascular and Microvascular Hemodynamics Assessed by Spectrophotometry in Periodontal Diseases
}

\author{
M. M. Figliuzzi $\mathbb{i D}^{1},{ }^{1}$ S. Sestito, ${ }^{1}$ D. Pacifico, ${ }^{1}$ L. Parentela, ${ }^{1}$ and Carlo Rengo ${ }^{2}$ \\ ${ }^{1}$ Medicine and Surgery School, Department of Health and Oral Sciences Periodontology Clinic, \\ "Magna Graecia" University of Catanzaro, Catanzaro, Italy \\ ${ }^{2}$ University of Naples "Federico II", Napoli, Italy
}

Correspondence should be addressed to M. M. Figliuzzi; figliuzzi@unicz.it

Received 21 March 2021; Revised 24 May 2021; Accepted 28 May 2021; Published 4 June 2021

Academic Editor: Gaetano Isola

Copyright $\odot 2021$ M. M. Figliuzzi et al. This is an open access article distributed under the Creative Commons Attribution License, which permits unrestricted use, distribution, and reproduction in any medium, provided the original work is properly cited.

Objective. The aim of this study is to identify a possible link between macrovascular hemodynamic status and microvascular hemodynamic indices in patients with periodontal disease. Methods and Materials. Seventeen adult patients are recruited on a voluntary basis at the Dentistry Department of the "Mater Domini" University of Catanzaro, with sampling that determines the lipid profile, blood glucose, inflammatory mediators, blood plasma viscosity: anamnesis, blood pressure measurement, and detection of anthropometric parameters: eco-Doppler of the carotid arteries and brachial arteries with noninvasive measurements of hemodynamics and evaluation of inflammation and periodontal circulation with a noninvasive spectroscopic technique. The subjects underwent a dental inspection with periodontal proves. The different indices of periodontal disease were evaluated. Results. The sites with high probing depth differ from the healthy ones, showing low oxygen saturation and a notable increase in tissue edema, but no correlation between macro- and microvascular values was found. Conclusion. Periodontal probing and spectroscopic examination showed the correlation between low oxygen saturation levels and tissue edema values with probing depth; however, no correlation between macrovascular hemodynamic status and microvascular hemodynamics indices was found probably given the heterogeneity of the population under consideration, the low number of data gathered, and the small sample size.

\section{Introduction}

The association between dental and cardiovascular diseases has been arousing much interest in the recent years.

Some authors have investigated the correlation between the levels of some chronic inflammation mediators present in the blood and saliva and the severity of periodontal disease.

Recently a study wanted to analyze and identify the association among salivary interleukin-6 (IL-6) levels and periodontitis (PT) and to determine the significant trend of this association in PT patients.

Patients in the PT group had significantly higher median salivary IL-6 levels compared to the HS group. Salivary IL-6 levels were negatively correlated with C-reactive protein, with the number of teeth and with clinical attachment loss $(\mathrm{CAL})$, probing pocket depth (PPD), and bleeding sites (FMBS). Furthermore, in patients with PT, salivary IL-6 levels were inversely associated (P-trend) with the number of teeth, and directly associated with the proportional extent of PT; these results so showed that PT patients presented significant higher salivary IL-6 levels compared to HS. Moreover, in the analyzed sample, a significant p-trend among PT, tooth loss, and increased salivary IL-6 levels was found [1].

Another study from the same group of authors aimed to analyze the association and impact of periodontitis and coronary heart disease (CHD) on salivary and serum galectin-3 in patients with periodontitis and CHD; the results showed that patients with periodontitis and 
periodontitis + CHD presented significant higher serum and salivary galectin-3 levels in comparison with CHD patients and healthy subjects. Periodontitis and ET-1 were the significant predictors of serum and salivary galectin-3 levels, respectively. [2].

Another study found increased leukotriene concentration in the gingival crevicular fluid from subjects with periodontal disease and atherosclerosis. [3].

Various studies have reported that there is a correlation between CHD risk factors or metabolic syndrome and related diseases (arterial hypertension, obesity, or diabetes) and periodontal disease, and this is very interesting due to the potential clinical implications $[4,5]$.

Hemodynamics is that branch of cardiovascular physiology that analyzes and studies the behavior of blood moving in the vessels, therefore allowing reducing the danger of forming pathologies related to blood circulation.

The functioning of the cardiocirculatory system can be explained using the physical principles derived from the laws of hydrostatics-hydrodynamics, although the cardiocirculatory system has features that prevent a precise quantitative description.

In fact, the cardiocirculatory system presents

(i) elastic and nonrigid ducts

(ii) tracts (capillaries) that allow the leakage and the entry of liquids

(iii) a pump (heart) with intermittent activity

(iv) external pressure in the ducts which can determine changes in the caliber due to its elasticity

(v) a non-Newtonian fluid in motion (blood), with viscosity varying with variation of speed

The blood is a complex fluid consisting of a suspension of particles in liquid form (plasma) and, as such, subject to variations in viscosity. The blood viscosity depends on the temperature and hematocrit. Usually, we consider the blood viscosity to be around $4.103 \mathrm{~kg} \mathrm{~m}^{-1} \mathrm{~s}^{-1}$ at a temperature of $37^{\circ} \mathrm{C}$ and a hematocrit of 45 . Basically, the viscosity can be considered as a resistance in flow, given that the layers of fluid subjected to intermolecular attractions oppose a deformation.

The monitoring of hemodynamic forces in patients with periodontal disease could be important for an accurate evaluation of the overall cardiovascular risk for periodontal therapy [6].

The wall shear stress is the frictional force that is generated by the blood when flowing tangentially to the endothelial surface, strongly influencing the arterial remodeling and, if low, promotes the formation of plaques.

It is known that the wall shear stress is lower in elder people and in those with high blood pressure, high body mass index, or affected by diabetes $[7,8]$. Moreover, the wall shear stress is generally different between males and females as the periodontal disease, tooth loss, and atheroschlerosis [9]. Furthermore, this association is independent of the classic CHD risk factors and age. It has been proved that the wall shear stress decreases with the worsening of periodontal health, potentially causing atherogenic local flow conditions.
The way in which periodontitis affects hemodynamics could be the systematic inflammation [10], with an enlargement in arterial diameter causing, for example, rheumatoid arthritis. Several studies have proven that inflammatory mediators such as interleukins and TNF- $\alpha$, produced by the inflamed oral tissues [11], can activate systemic inflammation and the subsequent immune cascade, with the resulting low-grade chronic inflammation which should promote the development of atherosclerotic disease [12]. Furthermore, a low shear stress could in turn increase the local vascular inflammation.

The calculation of the hemodynamic forces is based on the eco-Doppler measurements: this method is fast, repeatable, of low cost, valid, and vastly applied in this field.

The aspect investigated in this study is the association between local carotid hemodynamics, microvascular hemodynamics indices, and periodontal disease, and the purpose is to identify the link between periodontal indices and micro- and macrovascular hemodynamics values, in particular the wall shear stress, suggesting that the alternation of the hemodynamic profile could contribute to atherosclerosis in subjects with periodontal disease.

\section{Methods and Materials}

Seventeen adult patients who meet the inclusion criteria are sequentially recruited on a voluntary basis at the dentistry crime of the "Mater Domin" University Hospital of Catanzaro; those who meet the inclusion criteria signed written consent.

\subsection{The Inclusion Criteria}

(i) Patients with periodontal disease requiring dental therapy (moderate or advanced periodontal lacerations)

\subsection{The Exclusion Criteria}

\section{(i) Atrial fibrillation}

(ii) Significant hemodynamically carotid stenosis

(iii) Use of drugs with an antihypertensive effect

(iv) Nonmenopausal women or neoplasm in act

Informative consent is signed by each subject before the spectra are collected. The periodontal sites are defined as those with periodontal probing depth $\geq 5 \mathrm{~mm}$ and a loss in clinical attachment of $\geq 3 \mathrm{~mm}$. Gingivitis sites are defined as those with periodontal probing depth $<3 \mathrm{~mm}$ and bleeding on probing. Healthy sites are defined as those with probing depth $<3 \mathrm{~mm}$ and no bleeding on probing. The spectra were obtained from the contralateral healthy sites or the closest contralateral site closest to the diseased site. The spectra measurements were acquired in the following site: MeaBuccal, Mid-Lingual, Mesio-Buccal, Mesio-ingual, DistoBuccal and Disto-Lingual. 
The observational study clinic is based on the addition of a cardiovascular screening along with macro- and microvascular hemodynamic measures of selected dental patients. It is made up of

(i) blood sampling in order to determine the lipid profile, glycemia, inflammatory mediators, and blood and plasma viscosity

(ii) anamnesis, blood pressure measurement, and detection of anthropometric parameters

(iii) eco-Doppler ultrasound of the brachial carotid arteries with noninvasive measurement of hemodynamics

(iv) evaluation of the inflammation and periodontal circulation with a noninvasive spectroscopic technique

Systolic and diastolic blood pressures were measured by using a standardized sphygmomanometer on the right arm of the participants, after having rested for at least 5 minutes. An average of the second and third reading is then calculated.

The observational clinic study is, therefore, based on the addition of cardiovascular screening and of macro- and microvascular hemodynamic measures towards selected dental patients. It consists of blood sampling in order to determine the lipid profile, glycemia, and inflammatory mediators with blood and plasma viscosity; anamnesis, blood measurement, and detection of anthropometric parameters; Doppler ultrasound of the carotid and brachial arteries with noninvasive measurements of hemodynamics; and evaluation of inflammation and periodontal circulation with a noninvasive spectroscopic technique. Weight and height are measured with routine methods. The body mass index (BMI) was calculated as weight $(\mathrm{kg})$ divided by the height squared. Patients with values higher than $29.9 \mathrm{~kg} 7 \mathrm{~m} 2$ were considered to be obese. Glucose and lipids present in the blood were measured with kits available on the market. The eco-Doppler exam was conducted with an ATL 3000 activated ECG instrument equipped with a 5-0 MHz multifrequency linear probe.

The common carotid artery, the carotid bulb, and the internal and external carotid artery have all been studied longitudinally and transversally, with anterior, lateral, and posterior approaches. Each segment has been classified as normal, with plaque, and with stenosis. A segment with plaque showed a localized laceration which invaded the lumen by $>1,3 \mathrm{~mm}$, without enlarging the spectral or only in the deceleration phase of the systole, with systolic and peak flow velocity of $<120 \mathrm{~cm} / \mathrm{s}$. The segments with stenosis showed a spectral enlargement during the systole or a peak flow in systolic velocity of $<120 \mathrm{~cm} / \mathrm{s}$ (Table 1 ).

The peak and mean of the wall shear stress were measured in the common carotid $1-2 \mathrm{~cm}$ next to the bulb with the following formulas:

(i) Peak: $4 \eta$ VSPIIDT

(ii) Average: $4 \eta \mathrm{VM} \backslash \mathrm{IDR}$

Subjects underwent dental inspection with periodontal probes.
TABLE 1: Parameters used in formulas.

\begin{tabular}{lc}
\hline ID & Internal diameter; distance from the near and distant \\
lumen-intimate interface measured with the echography \\
IDR & Measured at time $R$ of the ECG \\
IDT & Measured at time T of the ECG \\
VSP & Peak systolic velocity \\
VM & Mean systolic velocity \\
\hline
\end{tabular}

(i) The gingival plaque was calculated using the Silness and Loe plaque index, while the gingival inflammation was assessed using the gingival index.

(ii) The depth of the pocket is evaluated. The gingival margin was taken as reference point when reading the values during probing.

As it is known, the periodontal indices are usually a sum of each dental element score, divided by the number of teeth, resulting in an average value for the patient. In this work, the sum of the values was calculated for each periodontal index of each patient. The staff performing the tests was unaware of the other measurements.

\section{Results}

The 17 patients in this preliminary phase presented an age range with varied from 23 to 74 years with an average of 49.8 years. The group was made up of 9 males and 8 females. Looking at the comparison between the periodontal probing and the spectroscopic examination, as already occurred in previous works of Liu $[13,14]$, the correlation between low oxygen saturation levels and the tissue edema values with the depth of the pol was confirmed. In fact the sites with gingivitis and the healthy sites presented superimposable spectra, while on the other hand, sites that presented an extensive depth of probing differed greatly from the healthy sites, highlighting the low oxygen saturation and a notable increase in the tissue edema. The hemodynamic and clinical results are presented in Table 2 .

The variability in measurements of macro- and microvascular hemodynamics was not correlated in the population under examination $(p>0.1)$.

The statistical analysis applied was a multiple linear correlational analysis made with SPSS v.17.0.

\section{Discussion}

The comparison between periodontal probing and spectroscopic examination showed, as in the past, the correlation between low oxygen saturation levels and tissue edema values with probing depth; in fact, the sites with high probing probability differ from the healthy ones, showing a low oxygen saturation and a notable increase in tissue edema. However, no correlation between macrovascular haemodynamic status and microvascular haemodynamic indices was found in the small population under examination. The negative result of the study could be attributed to numerous reasons. The most likely hypothesis is that, also given the heterogeneity of the population under consideration, the low number of data gathered affected the result. 
TABLe 2: Hemodynamic and clinical results.

\begin{tabular}{lcc}
\hline Parameters & Average or percentage & Standard deviation \\
\hline Age (years) & 49.8 & 15.4 \\
Peak_shear_stress carotid $\left(\right.$ dynes $\left./ \mathrm{cm}^{2}\right)$ & 25.3 & 6.5 \\
Mean shear carotid $\left(\right.$ dynes $\left.\mathrm{cm}^{2}\right)$ & 10.2 \\
Tissue oxygen saturation & 27.1 \\
Tissue water content & 11.3 \\
Obesity (\%) & 18 \\
Dislipidemia (\%) & 12 \\
Hyertention (\%) & 18 \\
Diabetes (\%) & 0 \\
Smoke (\%) & 35 \\
Alcohol (\%) & 18 \\
Cardiovascular diseases (\%) & 6 \\
Pathological carotid eco-Doppler (\%) & 6 \\
\hline
\end{tabular}

The statistic test used should correct these factors but probably not with great effectiveness given the small sample size. The other hypothesis is that the spectrophotometric measurements, indirect for hemodynamics, were represented very little by the gingival microcirculation for there to be a strong and, therefore, visible association with the already few macrovascular hemodynamics. Finally, it is possible that the two circulatory districts were not strongly connected in the gingival pathology, and due to the enlargement of the local gingivopathy, it was only the systematic inflammation which gave the verified worsening of the carotid hemodynamic situation in patients with periodontal disease.

\section{Data Availability}

The data are private as they are of patients of the Mater Domini University Hospital; they can be obtained on request from the corresponding author.

\section{Conflicts of Interest}

The authors declare that they have no conflicts of interest.

\section{References}

[1] G. Isola, A. Lo Giudice, A. Polizzi, A. Alibrandi, P. Murabito, and F. Indelicato, "Identification of the different salivary Interleukin- 6 profiles in patients with periodontitis: a crosssectional study," Archives of Oral Biology, vol. 122, Article ID 104997, 2021.

[2] G. Isola, A. Polizzi, A. Alibrandi, R. C. Williams, and A. Lo Giudice, "Analysis of galectin-3 levels as a source of coronary heart disease risk during periodontitis," Journal of Periodontal Research, vol. 56, 2021.

[3] M. Bäck, S. Airila-Månsson, T. Jogestrand, B. Söder, and P.-Ö. Söder, "Increased leukotriene concentrations in gingival crevicular fluid from subjects with periodontal disease and atherosclerosis," Atherosclerosis, vol. 193, no. 2, pp. 389-394, 2007.

[4] C. Carallo, M. S. D. Franceschi, C. Tripolino et al., "Periodontal treatment elevates carotid wall shear stress in the medium term," Medicine, vol. 94, no. 42, Article ID e1724, 2015.
[5] C. Carallo, L. Fortunato, M. S. de Franceschi et al., "Periodontal disease and carotid atherosclerosis: are hemodynamic forces a link?" Atherosclerosis, vol. 213, no. 1, pp. 263-267, 2010.

[6] C. Carallo, M. S. De Franceschi, C. Tripolino et al., "Common carotid and brachial artery hemodynamic alterations in periodontal disease," Journal of Clinical Periodontology, vol. 40, no. 5, pp. 431-436, 2013.

[7] C. Irace, C. Carallo, A. Crescenzo et al., "NIDDM is associated with lower wall shear stress of the common carotid artery," Diabetes, vol. 48, no. 1, pp. 193-197, 1999.

[8] S. K. Samijo, J. M. Willigers, R. Barkhuysen et al., "Wall shear stress in the human common carotid artery as function of age and gender," Cardiovascular Research, vol. 39, no. 2, pp. 515-522, 1998.

[9] M. Desvarieux, C. Schwahn, H. Völzke et al., "Gender differences in the relationship between periodontal disease, tooth loss, and atherosclerosis," Stroke, vol. 35, no. 9, pp. 2029-2035, 2004.

[10] N. M. Moutsopoulos and P. N. Madianos, "Low-grade inflammation in chronic infectious diseases: paradigm of periodontal infections," Annals of the New York Academy of Sciences, vol. 1088, pp. 251-264, 2006.

[11] E. De Nardin, "The role of inflammatory and immunological mediators in periodontitis and cardiovascular disease," $A n$ nals of Periodontology, vol. 6, no. 1, pp. 30-40, 2001.

[12] C. Irace, G. Mancuso, E. Fiaschi, A. Madia, G. Sesti, and A. Gnasso, "Effect of anti TNFalpha therapy on arterial diameter and wall shear stress and HDL cholesterol," Atherosclerosis, vol. 177, no. 1, pp. 113-118, 2004.

[13] K. Z. Liu, X. M. Xiang, A. Man et al., "In vivo determination of multiple indices of periodontal inflammation by optical spectroscopy," Journal of Periodontal Research, vol. 44, no. 1, pp. 117-124, 2009.

[14] X. Xiang, M. G. Sowa, A. M. Iacopino et al., "An update on novel non-invasive approaches for periodontal diagnosis," Journal of Periodontology, vol. 81, no. 2, pp. 186-198, 2010. 